\title{
Ground state octupole correlation energy with effective forces
}

\author{
L.M. Robledo 1, * \\ ${ }^{1}$ Departamento de Física Teórica, Universidad Autónoma de Madrid, E-28049 Madrid, Spain
}

\begin{abstract}
The ground state octupole correlation energy is computed with the D1M variant of the Gogny force in different theoretical frameworks and analyzed in detail. First I consider the correlation energy gained at the mean field level by breaking reflection symmetry. Next I consider the energy gain coming from symmetry (parity) restoration and finally I analyze the ground state correlation energy after configuration mixing with axially symmetric octupole states. The impact of the latter on theoretical binding energies indicates that octupole correlations do not affect in a significant way the trend and systematic of binding energies and therefore can not improve the performance of theoretical models in this respect. In particular, the too-large "shell gaps" predicted by selfconsistent mean field models and relevant in astrophysics scenarios are not altered by the octupole correlations.
\end{abstract}

\section{INTRODUCTION}

Many physical applications require a precise and accurate knowledge of nuclear binding energies. A typical example is found in the area of astrophysical simulations used to describe explosive scenarios where the goal is to understand how chemical elements heavier than iron are formed in the Universe [1-3]. The nuclear reactions involved take place along the "r-process" path that is located in regions of neutron rich nuclei well away from the stability line. Those nuclei are not within experimental reach in the present day or near future experimental facilities [4] and therefore astrophysical simulations require as input theoretical nuclear structure data. A consistent framework working with the same accuracy all over the nuclide chart, as required by astrophysical simulations, necessarily involves mean field techniques with effective interactions [5]. For the latter it is customary to used the family of Gogny forces [6] focusing on the D1M parametrization [7] for its good reproduction of nuclear binding energies. All the Gogny forces are characterized by a finite range central potential linear combination of gaussian shapes with different ranges and the standard combination of spin, isospin and spatial exchange terms. A standard zero range spin-orbit term and the Coulomb potential constitute the rest of the nuclear potential. The force is supplemented by a zero range density dependent term used to enforce the saturation mechanism of atomic nuclei. The parameters of the different Gogny forces are fitted using different strategies. In the case of D1M the parameters were fitted to reproduce the binding energies of all known nuclei computed with the Hartree- Fock- Bogoliubov (HFB) method [8] supplemented with an approximate rotational energy correction and an approximate zero point energy correction from quadrupole motion in the spirit of the 5D Bohr hamiltonian. In this way an impressive rms deviation for the binding energy of $0.79 \mathrm{MeV}$ is obtained. Charge radii and realistic symmetric and neutron mat-

\footnotetext{
* luis.robledo@uam.es http://gamma.ft.uam.es/robledo
}

ter equation of state were also used as target quantities in the fit. The specific values of the D1M parameters are given in Ref [7]. Recently, additional correlation energies including Projection on Particle Number (PNP), exact angular momentum projection (AMP) of deformed axially symmetric states and quadrupole configuration mixing with the projected states have been computed with Gogny D1M [9]. Unfortunately none of these effects seem to improve the agreement with experimental data in a significant way at variance with other studies using Skyrme or Gogny forces [10, 11].

Next in the hierarchy of multipole moments describing the shape of the nucleus comes the octupole moment with its characteristic breaking of reflection symmetry (parity). The purpose of this paper is to explore the correlation energy associated with the axially symmetric octupole degree of freedom [12, 13] and its impact on binding energies. Contrary to the quadrupole case, where correlation energies reach tens of $\mathrm{MeV}$ already at the mean field level, the octupole ones never exceed 2.5 $\mathrm{MeV}$. This is a modest addition but can have a strong impact on astrophysical scenarios where an accuracy of a couple hundred $\mathrm{keV}$ is required [2].

This paper is a follow up of Ref [14] where a systematic calculation of octupole properties over a large set of even-even nuclei was carried out. In the accompanying material of [14] three tables with the relevant quantities obtained with three parametrizations of the Gogny force were included. In those tables the correlation energies were already included but never discussed. Given the renewed interest on nuclear binding energies [2, 3, 9] I consider now timely to discuss in detail the impact of octupole correlations in those quantities.

\section{METHODOLOGY}

The computational procedure is the same as in Ref [14] where a thorough account of the properties of negative parity excited states was given using the HFB, parity projection and configuration mixing methods. Therefore I will give here only a short description for the convenience of the reader. First, the HFB equation with a 


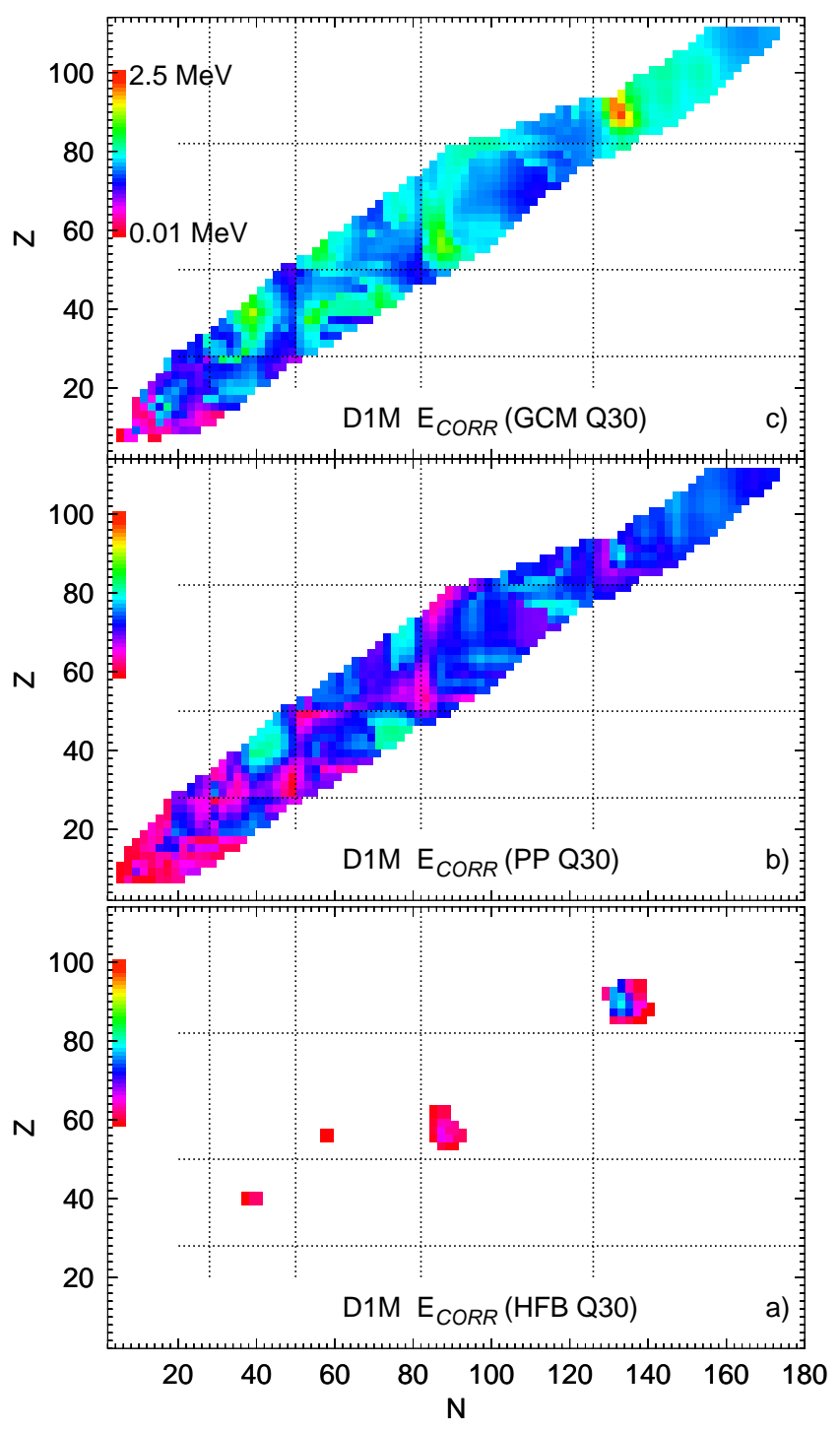

FIG. 1. (Color online) Color scale representation of the octupole correlation energy gain as compared to HFB results preserving reflection symmetry. In panel a) the HFB correlation energy gained by breaking reflection symmetry. In panel b) the parity RVAP correlation energy. In panel c) the octupole GCM correlation energy. Horizontal and vertical dotted lines correspond to magic proton and neutron numbers.

constraint on the axial octupole moment is solved using the approximate second order gradient method [15] with the HFBaxial computer code [16]. A set of axially symmetric HFB wave functions $\left|\Phi\left(Q_{30}\right)\right\rangle$ is generated this way. The interaction used is the Gogny force [6] with the D1M parametrization [7]. The location of the minimum of the HFB energy curve $E_{\mathrm{HFB}}\left(Q_{30}\right)$ determines whether the ground state is octupole deformed or not. The energy difference $\Delta E_{\mathrm{HFB}}=E_{\mathrm{HFB}}\left(Q_{30 \mathrm{~min}}\right)-E_{\mathrm{HFB}}(0)$ is the mean field correlation energy gained by the spontaneous breaking of reflection symmetry. In the next step, the octupole deformed HFB states are projected to good parity $\pi= \pm 1$ and the corresponding projected energies

$$
E_{\pi}\left(Q_{30}\right)=\frac{\left\langle\Phi\left(Q_{30}\right)\left|\hat{H} \hat{P}_{\pi}\right| \Phi\left(Q_{30}\right)\right\rangle}{\left\langle\Phi\left(Q_{30}\right)\left|\hat{P}_{\pi}\right| \Phi\left(Q_{30}\right)\right\rangle}
$$

are computed (paying special attention to the consistency problems associated with the use of density dependent forces [17, 18]). The minima of the two curves $E_{+}\left(Q_{30}\right)$ and $E_{-}\left(Q_{30}\right)$ at octupole moment values $Q_{30, \mathrm{PP}} \min$ and $Q_{30, \mathrm{NP} \text { min }}$, respectively, determine the optimal intrinsic state for each parity in the spirit of the restricted variation after projection (RVAP) method [19]. In eveneven nuclei the ground state always has positive parity and the RVAP correlation energy is given by $\Delta E_{\mathrm{RVAP}}=$ $E_{+}\left(Q_{30, \mathrm{PP} \min }\right)-E_{\mathrm{HFB}}(0)$. Finally, a Generator Coordinate Method (GCM) calculation is performed using the set $\left|\Phi\left(Q_{30}\right)\right\rangle$ of HFB wave functions as basis (generating coordinate) states. The solution of the Hill-Wheeler equation corresponding to the minimum energy is by definition the ground state, that has positive parity in eveneven nuclei. The correlation energy is defined in this case as $\Delta E_{\mathrm{GCM}}=E_{\mathrm{GS} \mathrm{GCM}}-E_{\mathrm{HFB}}(0)$.

By considering the octupole degree of freedom alone, I am implicitly assuming that it is not coupled to other degrees of freedom like quadrupole deformation or pairing. Given the negative parity of this degree of freedom, the above assumption seems reasonable but it has yet to be confirmed. In similar GCM calculations coupling the quadrupole and octupole degree of freedom [20 23] this assumption seems to be fulfilled and, as a consequence, the ground state octupole correlation energy is an additive quantity.

Recently, non axial degrees of freedom $Q_{32}$ related to tetrahedral correlations have been considered by several authors both with Skyrme [24] and Gogny [25] forces. Their impact on ground state correlation energies over the whole nuclear chart and specially when angular momentum projection is considered [25] remains to be explored.

\section{RESULTS}

First a HFB calculation preserving reflection symmetry is carried out for a set of 818 even even nuclei from oxygen to copernicium. The choice is made as to include the 620 even-even nuclei with known binding energies as given in the 2012 binding energy compilation [27] plus a few more. The binding energy obtained this way is used as the the reference energy in the definition of the different correlation energies. In Fig 1 the different correlation energies computed according to the approaches described above are plotted using a color scale. The three approaches are the reflection symmetry breaking HFB, the parity RVAP and finally the GCM with $Q_{30}$ as collective coordinate. In panel a) the HFB results $\Delta E_{\mathrm{HFB}}$ are depicted: just a few nuclei in the $\mathrm{Ra}, \mathrm{Ba}$ and $\mathrm{Zr}$ regions show a non-zero correlation energy that never exceeds $1.2 \mathrm{MeV}$. Those are 


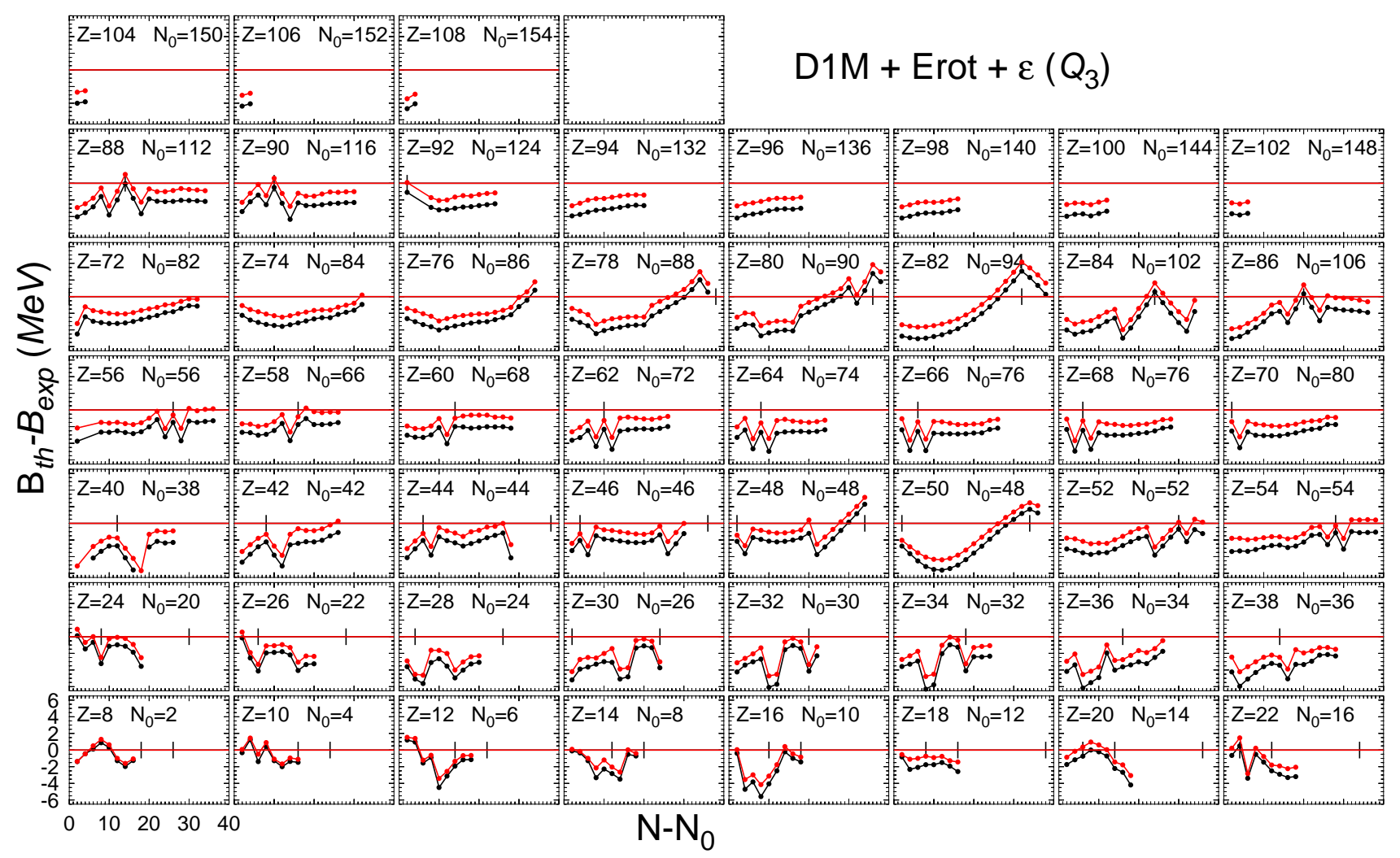

FIG. 2. (Color online) Binding energy difference between the theoretical predictions and the experimental data (2012 compilation [27]) plotted as a function of neutron number differences $N_{-} N_{0}$. The parameter $N_{0}(Z)$ is a reference neutron number specific of each $Z$ value. In each panel the corresponding values of $Z$ and $N_{0}(Z)$ are given in the label. The horizontal line correspond to a perfect agreement theory-experiment. The vertical lines found along this reference horizontal line signal magic neutron numbers. The black curves correspond to the theoretical binding energy computed with D1M using the HFB energy plus the rotational energy correction. The red curves additionally include the octupole correlation energy of the GCM calculation.

the regions where both proton and neutron numbers are close to the numbers favoring octupole correlations [12]. Parity projection on top of both reflection symmetric as well as octupole deformed HFB ground states produce a negligible energy gain as discussed in ref [14]. However, when parity RVAP is considered in addition to HFB, it leads to a non zero value of the octupole correlation energy in all the nuclei considered and as large as $1.5 \mathrm{MeV}$, as shown in panel b). The parity RVAP produces the largest correlation energy increase in octupole soft nuclei whereas the increase is essentially zero in those nuclei which are octupole deformed at the HFB level. The RVAP correlation energy shows some amount of shell effects as its value tend to be small close to magic proton and neutron numbers. Finally, the GCM correlation energy includes in its definition the symmetry breaking HFB correlation energy, the parity RVAP one as well as the correlation energy gained by the fluctuating octupole degree of freedom. This correlation energy is largest in those regions showing octupole deformation at the HFB level as observed in panel c) of Fig 1 . The GCM correlation energy can be as large as $2.5 \mathrm{MeV}$. In the regions in between the correlation energy is not as large and is typically of the order of $1 \mathrm{MeV}$ changing smoothly as a function of proton and neutron number and showing no indication of strong shell effects. At this point it can be concluded that ground state octupole correlation energies are not going to impact in a significant way the behavior of binding energies with proton or neutron number (only a roughly constant shift).

Similar results are obtained for the D1S and D1N [26] parametrizations of the Gogny force. The corresponding plots can be found in the accompanying material.

In order to understand the impact of the octupole correlation energy in the description of the binding energies we have plotted in Fig 2 the binding energy difference between two of the theoretical results and the experimental data (extracted from the 2012 compilation [27]). These quantities are plotted as a function of neutron number for each $Z$ value considered in the calculation (from oxygen to copernicium). In each panel two curves are shown: one corresponds to the HFB with octupole deformation plus rotational correction results and the other to the results including in addition the octupole GCM correlation energy. In most of the cases, the inclusion of the GCM octupole correlation energy represents a mere displace- 


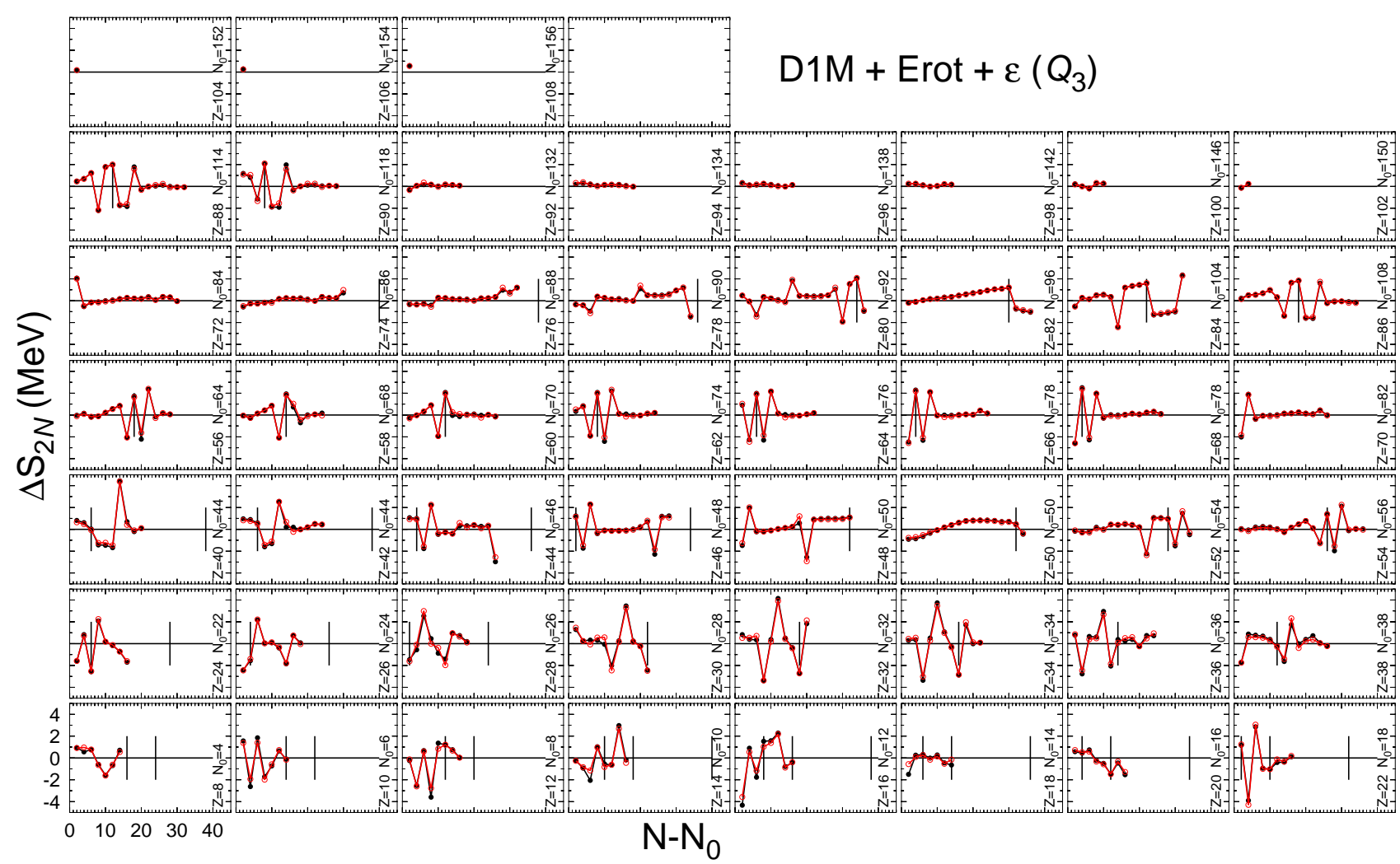

FIG. 3. (Color online) The difference between the theoretical prediction for the two neutron separation energy and the

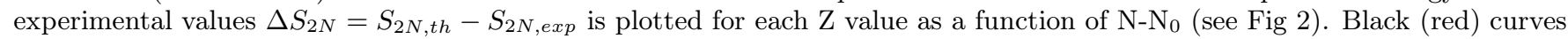
correspond to the results obtained with the HFB (GCM) theoretical approaches.

ment upwards of the curves and little improvement in the description of experimental data is observed. A comment is in order: in the calculations I do not have access to the zero point energy correction of the quadrupole degrees of freedom as computed in [7] and therefore this quantity has not been added to the binding energy. It is typically of the order of $2-3 \mathrm{MeV}$ and its neglect explains the overall shift observed in the D1M HFB predictions as compared to the experimental data. It is interesting to note that the largest discrepancies always take place around magic numbers and are specially remarkable for $Z=48-52$ and $Z=80-84$. In those cases the inclusion of the octupole correlation energy somehow reduces the discrepancy but is far from being enough to provide a good description of experimental data. When proton and neutron numbers are far from the magic numbers the behavior of the binding energy differences is quite smooth and almost independent of $N$ in most of the cases.

Another quantity connected to binding energies and of interest in astrophysics simulations is the two neutron separation energy $S_{2 N}$. The reaction rates of nuclear reactions depends on the $\mathrm{Q}$ value which is related to the $S_{2 N}$. At magic neutron numbers this quantity shows a sudden drop with its magnitude known as the "shell gap". The magnitudes of the shell gap for each proton number strongly influence the creation rate of heavy elements in nucleosynthesis environments. The shell gaps are not well reproduced by HFB mass models: before the drop of the $S_{2 N}$ at magic neutron numbers the theoretical models predict too high values for this quantity but the prediction becomes too small after the drop. As a consequence the mean field values of the shell gaps are predicted to be too large. The expectation is that correlations beyond the mean field will quench the "shell gap" (see 2] for a discussion in the astrophysical context) but the amount of quenching associated to each of the effects considered on top of HFB is still under debate [9]. To analyze the impact of octupole correlation energies on the shell gaps and the two-neutron separation energies the difference $\Delta S_{2 N}=S_{2 N, t h}-S_{2 N, \text { exp }}$ is plotted in Fig 3 in separated panels for each $Z$ value and as a function of $N$. As theoretical models I consider the HFB and the octupole GCM. To help reading the results the zero is marked by a horizontal line and vertical lines are placed at magic neutron numbers. The too large mean field shell gaps are responsible for the observed sudden change from positive to negative values when crossing magic neutron numbers. The conclusion extracted from the plot is that the extra correlation energies associated with the octupole GCM do not modify in a substantial way the HFB $\Delta S_{2 N}$ values (the two curves are almost indistinguishable for most $Z$ values) and therefore octupole correlations do not help to improve the agreement with experimental data for the $S_{2 N}$. As a consequence, 
octupole correlations do not quench the too large mean field "shell gaps".

The present discussion has focused on the results for the D1M parametrization of the Gogny force but similar conclusions can be drawn for the D1S and D1N parametrizations. In the accompanying material plots like the ones of Fig 1 but for D1S and D1N are included.

I would like to point out that the irrelevant role played by octupole correlation energies in the description of nuclear binding energies and the associated reaction $Q$ values by no means diminishes the importance of octupole correlations in the description of other aspects of nuclear structure like low lying negative parity states and the associated enhanced E3 transition strengths (see [12] for a thorough review including both phenomenology and theoretical methods, and [13] for a recent account), the physics of super-heavy nuclei [28, 29] or the description of alternating parity rotational bands [30, 31] just to cite a few.

\section{CONCLUSIONS}

The ground state octupole correlation energy defined at three levels of approximation: the octupole deformed HFB, the parity RVAP and the octupole GCM are computed with the Gogny D1M effective interaction. Although non negligible and as large as $2.5 \mathrm{MeV}$, the octupole GCM correlation energy does not improve substantially the agreement between theory and experiment for binding energies. This is a consequence of the rather smooth behavior of the correlation energy as a function of $Z$ and $N$. The impact on the "shell gaps" is also imperceptible. I conclude that the ground state octupole correlation energy only contributes to minor improvements in the description of binding energies and plays a minor role in astrophysical simulations.

\section{ACKNOWLEDGMENTS}

Work supported in part by Spanish MINECO grants Nos. FPA2012-34694 and FIS2012-34479 and by the Consolider-Ingenio 2010 program MULTIDARK CSD2009-00064.
[1] M. Arnould, S. Goriely, and K. Takahashi, Phys. Rep. 450, 97 (2007).

[2] A. Arcones and G. F. Bertsch, Phys. Rev. Lett. 108, 151101 (2012).

[3] R. Reifarth, C. Lederer, and F. Kappeler, J. Phys. G: Nucl. Part. Phys. 41053101 (2014).

[4] K. Blaum, Phys. Rep. 425, 1 (2006).

[5] M. Bender, P.-H. Heenen, P.-G. Reinhard, Rev. Mod. Phys. 75, 121 (2003).

[6] J. Decharge and D. Gogny, Phys. Rev. C 21, 1568 (1980).

[7] S. Goriely, S. Hilaire, M. Girod, and S. Péru, Phys. Rev. Lett. 102, 242501 (2009).

[8] P. Ring and P. Schuck, "The Nuclear Many-Body Problem", (Springer, NY, 1980).

[9] T.R. Rodriguez, A. Arzhanov, G. Martinez-Pinedo, arXiv:1407.7699

[10] B. Sabbey, M. Bender, G.F. Bertsch, and P.-H. Heenen, Phys. Rev. C 75, 044305 (2007).

[11] J.-P. Delaroche, et al., Phys. Rev. C 81014303 (2010).

[12] P.A. Butler and W. Nazarewicz, Rev. Mod. Phys. 68, 349 (1996).

[13] L. P. Gaffney et al., Nature 497, 199 (2013).

[14] L. M. Robledo and G. F. Bertsch, Phys. Rev. C 84, 054302 (2011).

[15] L. M. Robledo and G. F. Bertsch, Phys. Rev. C 84, 014312 (2011).
[16] L.M. Robledo, HFBaxial code (2002).

[17] L.M. Robledo, Intl. J. of Mod. Phys. E 16, 337 (2007).

[18] L.M. Robledo, J. Phys. G 37, 064020 (2010).

[19] J.L. Egido and L.M. Robledo, Nucl. Phys. 524, 65 (1991).

[20] L.M. Robledo, J.L. Egido, B. Nerlo-Pomorska, and K. Pomorski, Phys. Lett. B201, 409 (1988).

[21] J. Meyer, P. Bonche, M. S. Weiss, J. Dobaczewski, H. Flocard, and P.-H. Heenen, Nucl. Phys. A 588, 597 (1995)

[22] R.R. Rodriguez-Guzman, L.M. Robledo, and P. Sarriguren, Phys. Rev. C 86, 034336 (2012).

[23] L.M. Robledo and P.A. Butler, Phys Rev C 88, 051302 (2013).

[24] K. Zberecki, P. Magierski, P.-H. Heenen, and N. Schunck Phys. Rev. C 74, 051302(R) (2006).

[25] S. Tagami, Y. R. Shimizu, and J. Dudek J. Phys. G: Nucl. Part. Phys. 42, 015106 (2015).

[26] F. Chappert , M. Girod and S. Hilaire, Phys. Lett. B 668, 420 (2008).

[27] M. Wang et al, Chinese Phys. C 36, 1603 (2012).

[28] H. Wang, H. Liu, F. Xu, and C. Jiao, Chinese Science Bull. 57,N15, 1761 (2012)

[29] Y.-S. Chen, Y. Sun, and Z. Gao, Phys. Rev. C 77, 061305(R) (2008)

[30] S. Zhu, R.V.F. Janssens, G.J. Lane et al, Phys. Lett. B 618, $51(2005)$

[31] E. Garrote, J.L. Egido, and L.M. Robledo, Phys. Rev. Lett. 80, 4398 (1998) 


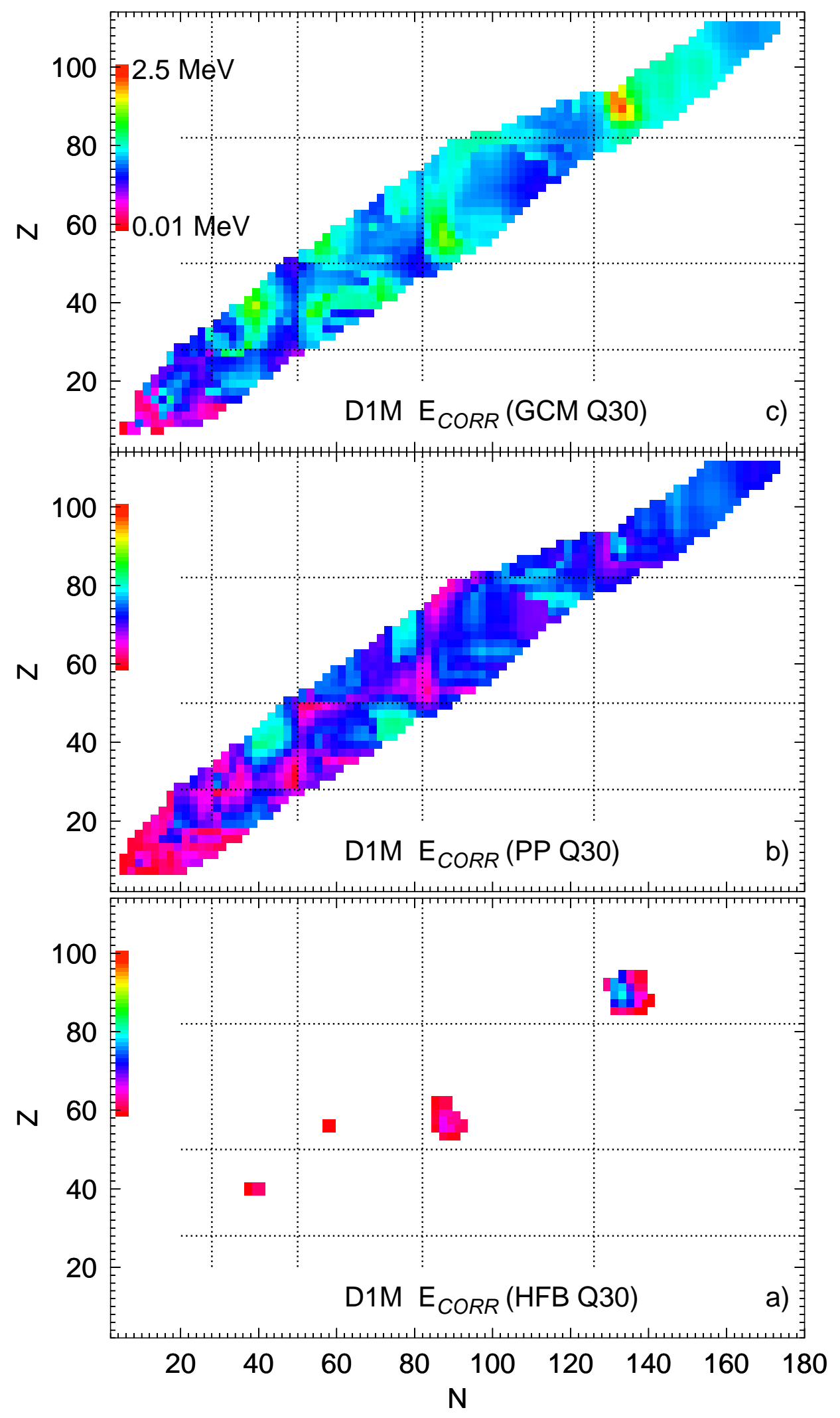




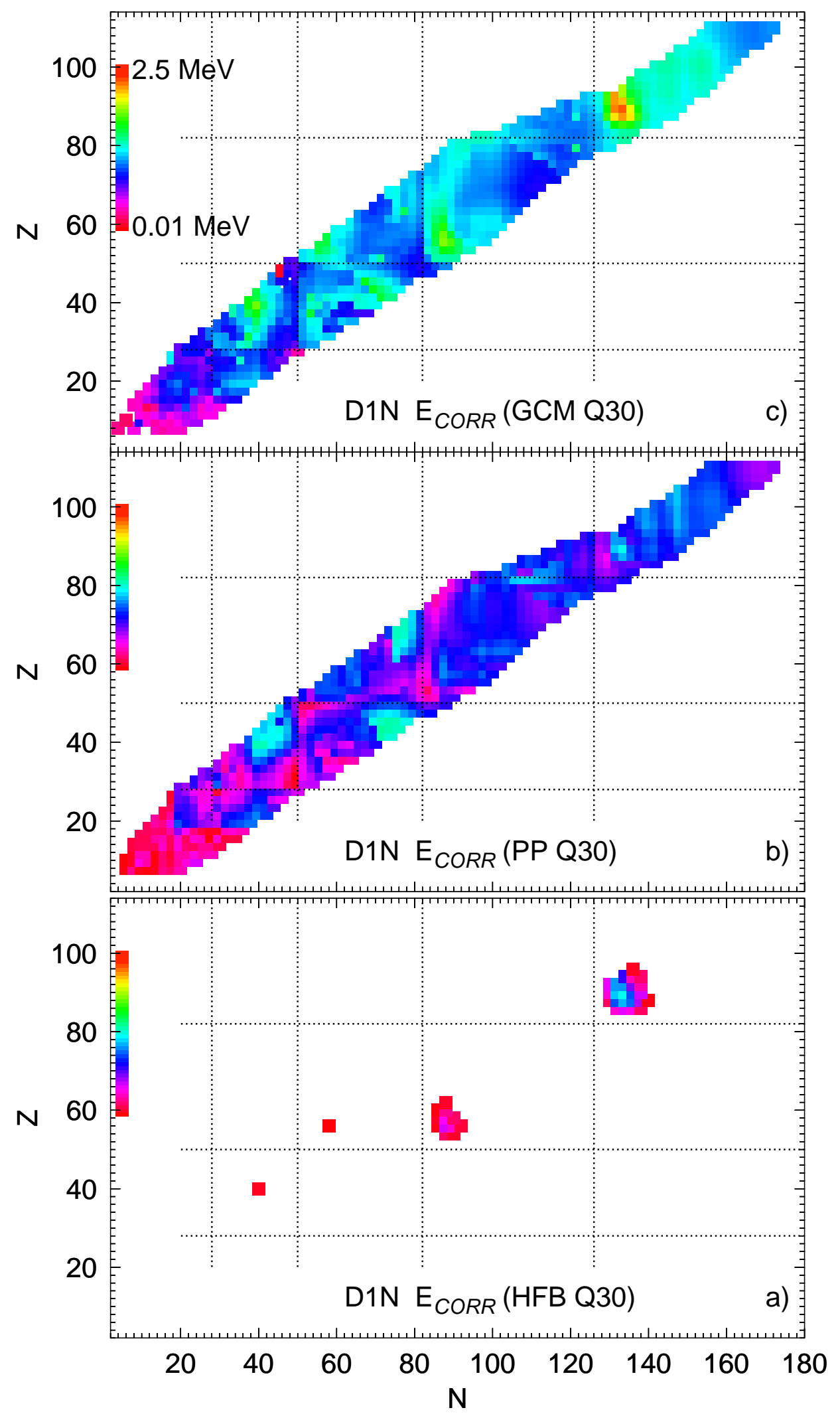




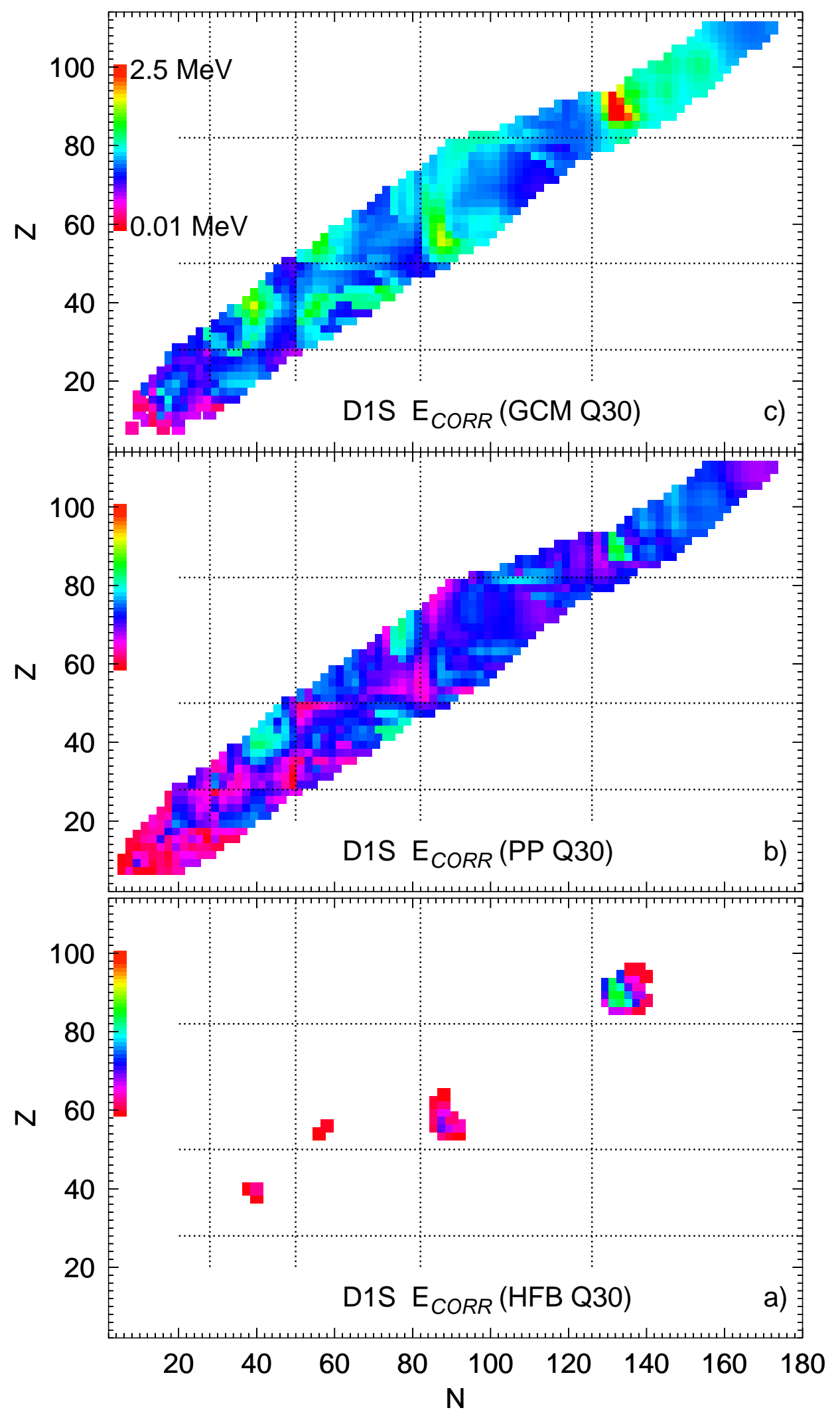

